2018;11:179.

2. Lee SWH, Lai NM, Chaiyakunapruk N, Chong DWK. Adverse effects of herbal or dietary supplements in G6PD deficiency: A systematic review. Br J Clin Pharmacol. 2017;83:172-9.
3. Schuurman M, Waardenburg DV, Costa JD, Niemarkt H, Leroy P. Severe hemolysis and methemoglobinemia following fava beans ingestion in glucose-6-phosphatase dehydrogenase deficiency - Case report and literature review. Eur J Pediatr. 2009;168:779-82.

\section{Acute Flaccid Myelitis: Are We Vigilant Enough?}

With the eradication of poliomyelitis from most countries, acute flaccid myelitis (AFM) due to non-polio enteroviruses and other viruses is an emerging disease. Besides the vaccineassociated paralytic polio, AFM outbreaks due to other viruses are also a hazard. AFM comprises of patients with acute flaccid paralysis (AFP), characteristically asymmetric limb weakness, with MRI suggestive of a spinal cord lesion in grey matter and spanning one or more vertebral segments [1]. Over the last decade, multiple outbreaks have been reported from countries such as USA, European countries, and Japan [1]. Two outbreaks have already been reported from India [2,3]. However, the pathogen testing was limited and inconclusive in both the cohorts. Even with ongoing AFP surveillance, AFM has not been frequently reported from India. Similar to Australia, we believe that there is misdiagnosis and underrecognition of AFM. During the initial disease course, AFM is frequently misdiagnosed as transverse myelitis due to an often extensive involvement of the spinal cord, not classically limited to the grey matter of the spinal cord [5]. Hence, there is a need for creating awareness regarding this evolving entity.

With many viruses involved such as EVD68, EVA71, etc. and poor yield of pathogen testing, it is often difficult to establish causality for AFM [1-3]. Therefore, it is time that patients with AFP should also be tested for other viruses beyond the poliovirus. This can later help in strengthening the AFP surveillance system. Survey studies for non-polio AFM throughout the country may be an initial step in this aspect, in the absence of active ongoing surveillance. However, the surveys need to be more robust to capture the epidemiological aspects of both AFM and associated respiratory/ gastrointestinal illnesses. The key epidemiological parameters should include the whereabouts of patients (for source identification), age group, details of neuroimaging, and virological studies, contact tracing, etc. for patients in both the groups. Besides, AFM clusters and outbreaks need to be investigated meticulously to avoid an epidemic staring at us.

Priyanka Madaan and Lokesh Saini* Pediatric Neurology Division, Department of Pediatrics, Advanced Pediatric Centre, PGIMER, Chandigarh, India.

*drlokeshsaini@gmail.com

\section{REFERENCES}

1. Hardy D, Hopkins S. Update on acute flaccid myelitis: Recognition, reporting, aetiology, and outcomes. Arch Dis Child. 2020;105:842-47.

2. Sarmast SN, Gowda VK, Ahmed M, Gv B, Saini J, Benakappa A. Acute flaccid myelitis - Clustering of poliolike illness in the tertiary care centre in Southern India. J Trop Pediatr. 2019;65:309-31.

3. Madaan P, Saini L. Nerve transfers in acute flaccid myelitis: A beacon of hope. Pediatr Neurol. 2019;93:68.

4. Madaan P, Keshavan S, Saini L. Poor clinico-radiological correlation: A hallmark of acute flaccid myelitis. Brain Dev. 2019;41:480-81.

\section{Acute Flaccid Paralysis in a Child: It Is Not Guillain-Barré Syndrome Always!}

A 6-year-old-girl presented with complaints of difficulty in walking for 5 days. Initially, the child started limping on the left side, followed by unable to bear weight; within two days, the right lower limb also got involved and she became nonambulatory. She also complained of dull aching pain in lower limbs, especially in the upper thigh, more on the left side. There was no history of proceeding febrile illness, trauma or intramuscular injection. She was completely immunized as per the national immunization schedule. She had tenderness in the left flank, lower back and bilateral thigh, keeping the hips in a semiflexed position, not allowing any passive movement or formal tone examination. Even knee jerks could not be elicited bilaterally. In the left hip joint power was $2 / 5$ and $3 / 5$ power in the right knee, left hip and knee joint. A clinical possibility of acute flaccid paralysis (AFP) was kept, with a differential diagnosis of Guillain-Barré syndrome (GBS), viral myositis, polymyositis, transverse myelitis, paralytic polio myelitis, Perthes disease, septic arthritis of the hip joint and pseudoparalysis due to unnoticed trauma, or with pelvis/femur fracture. On investigations, $X$-ray of the hips, nerve conduction study and serum creatine phosphokinase were normal. Ultrasonogram revealed a heterogeneous collection in left iliopsoas muscle, extending to the pelvis and inguinal region. Pus was drained by percutaneous pigtail catheter and she responded favorably to intravenous vancomycin and she was able to walk after three days. 